\title{
Erratum to: Constellations of Interpersonal Trauma and Symptoms in Child Welfare: Implications for a Developmental Trauma Framework
}

\author{
Cassandra L. Kisiel • Tracy Fehrenbach • \\ Elizabeth Torgersen • Brad Stolbach • Gary McClelland • \\ Gene Griffin • Kristine Burkman
}

Published online: 30 May 2014

(C) Springer Science+Business Media New York 2014

\section{Erratum to: J Fam Viol (2014) 29:1-14 \\ DOI 10.1007/s10896-013-9559-0}

The original version of this article unfortunately contained a mistake. The fourth paragraph under the subsection Symptom Clusters and Outcomes in the Results section is corrected as follows:

Further, in addition to showing an increased likelihood of developmental trauma symptom patterns, the complexly (violent/non-violent) traumatized youth in this sample, experienced significantly worse child welfare placement outcomes than did those with either violent or non-violent trauma exposure alone. Based on Zero-inflated Poisson regression analyses and Incident Rate Ratios, youth exposed to

both violent and nonviolent interpersonal trauma experienced 1.26 times (or $26 \%$ ) more placement disruptions in the 2 years following entry into care compared to those youth with other types of trauma $(p<.001)$. In comparison, youth exposed to non-violent trauma alone also experienced significantly more placement disruptions (by 1.15 times or $15 \%$ ) during this time frame $(p<.001)$; yet there was no significantly increased likelihood of placement changes for youth who had been exposed to violent trauma alone. The pattern is different for psychiatric hospitalization: the incident rate was 1.7 times higher for youth with violent/non-violent trauma $(\mathrm{p}<.001)$ and 1.37 times higher for youth with violent trauma $(p<.001)$ compared to youth with other types of trauma.

The online version of the original article can be found at http://dx.doi.org/ 10.1007/s10896-013-9559-0.

C. L. Kisiel $(\varangle) \cdot$ T. Fehrenbach · E. Torgersen • G. McClelland •

G. Griffin

Department of Psychiatry and Behavioral Sciences, Northwestern

University Feinberg School of Medicine, 710 N. Lake Shore Drive,

12th floor, Chicago, IL 60611, USA

e-mail: c-kisiel@northwestern.edu

B. Stolbach

Department of Pediatrics, The University of Chicago Priztker School

of Medicine, Chicago, IL, USA

K. Burkman

Department of Psychology, San Francisco VA Medical Center, San

Francisco, CA, USA 